\section{Pisa Biobank and Tuscany Regional Biorepository}

Francesca Nocchi, Patrizia Urciuoli, Silvia Ursino, Elisa Biagi, Simone Lapi

Departmental Section of Biobanks, Cisanello Hospital, Azienda Ospedaliero Universitaria Pisana, Pisa, Italy

\begin{abstract}
Departmental Section of Biobanks (SOD Biobanche) of the Azienda Ospedaliera Universitaria Pisana, consists of a Tissue and Cell institute, the Heart Valve Bank, and a biorepository, the Regional Biologic Archive. A clean room consisting of three laboratories (1 class C and 2 class B) represents a core facility for some of the processes and projects of the biobank. The Regional Biological Archive is a biobank which currently carries out as an institutional task the storage of serum, plasma and non-viable cells of multiorgan, multi-tissue donors and donors of corneas, as well as solid organ transplant recipients of the Tuscany Region, providing the possibility of reviewing and re-evaluating even at a distance of years the donor/recipient pairs The mission of SOD Biobanche is to act as a transversal service platform in the field of biobanking of biological material. In this broader perspective the structure is open to new projects concerning biobanking, some of which are already in the activation phase, such as a bank for urogenital tissues, a biobank of tumors of the musculoskeletal system and an advanced therapy protocol for the use of partially osteoinduced expanded mesenchymal stem cells.
\end{abstract}

\section{Introduction}

The common definition of biobank is "non-profit service unit for the collection, processing, storage and distribution of biological samples for research and diagnosis. We can identify different types of biobanks that show substantial differences both in terms of preserved material and legislation. However, there is a wide range of characteristics that represent a common denominator, starting from a quality assurance system.

\section{Results}

Departmental Section of Biobanks (SOD Biobanche) of the Azienda Ospedaliera Universitaria Pisana, consists of a Tissue and
Cell institute, the heart valve bank, and a biorepository, the Regional Biologic Archive. A clean room consisting of three laboratories ( 1 class $\mathrm{C}$ and 2 class $\mathrm{B}$ ) communicating through classified passage areas (Air lock) represents a core facility for some of the processes and projects of the biobank like tissue processing; facilities consists also of a sample acceptance, handling and sample rooms and a cryogenic area in a separate room monitored h24 where 15 cryogenic nitrogen containers are currently allocated and managed via software. Access to this structure (transport, acceptance, type and quantity of samples), included in the Tuscany Region transplantation network, is regulated by specific regional regulations and standard operating procedures. All processes are managed through a Quality Assurance System. The structure is ISO 9001 certified and accredited (tissue and cell institute) by the National Transplant Center (CNT).

The Regional Biological Archive is a biobank, established with DGR 1223/2004, which currently carries out as an institutional task the storage of serum, plasma and nonviable cells of multi-organ, multi-tissue donors and donors of corneas, as well as solid organ transplant recipients of the Tuscany Region. The inclusion of the Regional Biological Archive within a highly specialized and nationally relevant hospital has primarily represented the response to a need to implement the safety of the transplant network, providing the possibility of reviewing and re-evaluating even at a distance of years the donor/recipient pairs under the infectious profile.

Currently, over 160,000 plasma serum and non-vital lymphocytes are stored and cryopreserved in nitrogen vapors at $\mathrm{T}<-150{ }^{\circ} \mathrm{C}$ and at $-80^{\circ} \mathrm{C}$ in controlled freezers; the current flow stands at about 1200 aliquots of cryopreserved biological material/month. The biological material and the relative paper data are kept for 30 years. Complete traceability of biological material is guaranteed from the moment of acceptance until release. Each sample is uniquely identified through three different codes (internal ID, unique serial number automatically generated by the program, unique datamatrix code printed on the vial). The data are automatically backed up each time the session is closed and the data stored on the dedicated server located in the UOSI building of the Azienda Ospedaliero Universitaria Pisana.

Data management (bar code, personal data, internal ID, etc.) takes place with the help of a specific database (Freezerworks ${ }^{\circledR}$ unlimited), organized according to a client-server architecture. It is a validated software, which meets the FDA criteria cfr 21. The program allows the complete management of an unlimited number of containers (cryotanks, freezers) which can be configured according to the
Correspondence: Simone Lapi, Departmental Section of Biobanks, Cisanello Hospital, Azienda Ospedaliero Universitaria Pisana, Pisa, Italy.

E-mail: s.lapi@ao-pisa.toscana.it

Key words: Tissue establishment; regional biorepository; multispecialistic biobank.

Conference presentation: this paper was presented at the Second Centro 3R Annual Meeting - 3Rs in Italian Universities, 2019, June 20-21, University of Genoa, Italy.

Received for publication: 28 October 2019.

Accepted for publication: 6 November 2019 .

This work is licensed under a Creative Commons Attribution NonCommercial 4.0 License (CC BY-NC 4.0).

CCopyright: the Author(s), 2019

Licensee PAGEPress, Italy

Biomedical Science and Engineering 2019; 3(s2):92 doi:10.4081/bse.2019.92

user's specifications (number and type of rack, dimensions, position), for the purpose of defining unambiguously the position of the sample.

Parallel to the computerized archive, a paper archive is created containing the data of the donor / recipient. For the storage of the biological material, special vials of cryoresistant polypropylene are used (validated for use in nitrogen vapors) and with a unique twodimensional datamatrix code printed on the base (the company guarantees the uniqueness of each code stamped on each vial), indelible, specifically tested to resist abrasion, chemical insults and cryogenic temperatures $\left(<-150^{\circ} \mathrm{C}\right)$. Each vial is labeled using labels specifically validated for use in nitrogen vapors. The vials are lined up in special stainless steel containers and immersed in cryogenic containers fed with liquid nitrogen from a "continuous" line or, alternatively in controlled freezers at $-80^{\circ} \mathrm{C}$, and monitored through management software and an alarm system.

\section{Conclusions}

The mission of SOD Biobanche is to act as a transversal service platform in the field of bio-banking of biological material. In this broader perspective the structure is open to new projects concerning biobanking, some of which are already in the activation phase, such as a bank for urogenital tissues, a biobank of tumors of the musculoskeletal system and an advanced therapy protocol for the use of partially osteoinduced expanded mesenchymal stem cells. 